Dom. Cien., ISSN: $2477-8818$

Vol. 4, núm. 2, abril, 2018, pp. 63-80

Inversión y financiamiento para el sector agrícola del Ecuador: aplicación de un modelo de regresión múltiple

DOI: $10.23857 /$ dc.v4i2.778

(c) $)(9)$

Ciencias económicas y empresariales

Artículo de investigación

\title{
Inversión y financiamiento para el sector agrícola del Ecuador: aplicación de un modelo de regresión múltiple
}
Investment and financing for the agricultural sector of Ecuador: application of a multiple regression model

\section{Investimento e financiamento para o setor agrícola do Equador: aplicação de um modelo de regressão múltipla}

\author{
Víctor X. Quinde-Rosales ${ }^{1}$ \\ vquinde@uagraria.edu.ec \\ Rina M. Bucaram-Leverone II \\ rbucaram@uagraria.edu.ec \\ Martha R. Bucaram-Leverone ${ }^{\text {III }}$ \\ mbucaram@uagraria.edu.ec \\ Francisco A. Quinde-Rosales IV \\ franciscoquinderosales@outlook.es
}

Recibido: 14 de octubre de 2017 * Corregido: 10 de noviembre de 2017 * Aceptado: 12 de febrero de 2018

I Magister en Economía Agraria, Economista Agrícola, docente de la Facultad de Economía Agrícola de la Universidad Agraria del Ecuador, Guayaquil, Ecuador.

II Magister en Economía Agraria, Economista Agrícola, Ingeniera en Gestión Empresarial Internacional, Docente de la Facultad de Economía Agrícola de la Universidad Agraria del Ecuador, Guayaquil, Ecuador.

III Magister en Docencia Superior, Diplomado Superior en Investigación Educativa, Economista Agrícola, Ingeniera Comercial, Docente de la Facultad de Economía Agrícola de la Universidad Agraria del Ecuador, Guayaquil, Ecuador.

Iv Economista Agrícola de la Facultad de Economía Agrícola de la Universidad Agraria del Ecuador, Guayaquil, Ecuador. 
Dom. Cien., ISSN: 2477-8818

Vol. 4, núm. 2, abril, 2018, pp. 63-80

\section{Resumen}

El presente artículo, presenta la evaluación de la contribución generada por el gasto público, la inversión extranjera directa y el crédito bancario en el PIB agrícola, en el periodo de análisis trimestral del 2005 al 2015. Se realizó atendiendo una metodología de tipo inductiva dentro de un paradigma empírico-analítico. Para el proceso de los datos se utilizaron estimaciones de parámetros y pruebas estadísticas que validaron el modelo de regresión múltiple propuesto. Los resultados demostraron que las variables más importantes en términos de contribución con financiamiento o inversión es el crédito de la banca pública y el gasto público respectivamente; estos por cada unidad monetaria que invierten en el sector agrícola producen cerca de ocho veces más su valor para beneficio del sector productor, lo que permitió entre sus conclusiones evidenciar que las fuentes más importantes para generar una inversión o financiamiento de la agricultura son el gasto público, la inversión extranjera directa y el crédito bancario público y privado, convirtiéndose estas con sus capitales en impulso para el crecimiento de este sector histórico.

Palabras clave: gasto público; inversión extranjera directa; crédito; inversión; financiamiento; modelo de regresión múltiple.

\section{Abstract}

This article presents the evaluation of the contribution generated by public expenditure, direct foreign investment and bank credit in agricultural GDP, in the period of quarterly analysis from 2005 to 2015. It was carried out according to an inductive type methodology within an empirical-analytical paradigm. For the process of the data, parameter estimates and statistical tests were used to validate the proposed multiple regression model. The results showed that the most important variables in terms of contribution with financing or investment are the credit of the public bank and the public expenditure respectively; these for each monetary unit that invest in the agricultural sector produce about eight times more its value for the benefit of the producing sector, which allowed among its conclusions to show that the most important sources to generate an investment or financing of agriculture are public expenditure, direct foreign investment and public and private bank credit, becoming these with their capitals a boost for the growth of this historic sector.

64 Vol. 4, núm. 2, abril, 2018, pp. 63-80

Víctor Quinde Rosales, Rina Bucaram Leverone, Martha Bucaram Leverone, Francisco Quinde Rosales 
Dom. Cien., ISSN: 2477-8818

Vol. 4, núm. 2, abril, 2018, pp. 63-80

Inversión y financiamiento para el sector agrícola del Ecuador: aplicación de un modelo de regresión múltiple

Keywords: Public expenditure; foreign direct investment; credit; investment; financing; multiple regression model.

\section{Resumo}

Este artigo apresenta a avaliação da contribuição gerada pelo gasto público, investimento estrangeiro direto e crédito bancário no PIB agrícola, no período de análise trimestral de 2005 a 2015. Foi realizada de acordo com uma metodologia tipo indutiva dentro um paradigma empírico-analítico. Para o processo dos dados, estimativas de parâmetros e testes estatísticos foram utilizados para validar o modelo de regressão múltipla proposto. Os resultados mostraram que as variáveis mais importantes em termos de contribuição com financiamento ou investimento são o crédito do banco público e o gasto público respectivamente; estes para cada unidade monetária que investem no setor agrícola produzem cerca de oito vezes mais seu valor para o benefício do setor produtor, o que permitiu em suas conclusões mostrar que as fontes mais importantes para gerar um investimento ou financiamento da agricultura são as despesas públicas. , investimento estrangeiro direto e crédito bancário público e privado, tornando-se estes com seus capitais um impulso para o crescimento deste setor histórico.

Palavras chave: despesa pública; investimento estrangeiro direto; credito investimento; financiamento; modelo de regressão múltipla.

\section{Introducción}

La agricultura es un sector que presenta vínculos con la historia del Ecuador, fue el que financio el nacimiento republicano y la principal fuente de ingresos del Ecuador a inicios de la república, antes de conocer la existencia de pozos petroleros en el territorio patrio. Del mismo modo es el sector productivo, que presenta una flexibilidad en términos de aportar productos para la exportación sin caer en la dependencia mono-exportadora y mono-productora que singularizó a los países latinoamericanos de inicio republicano. La producción agrícola alberga el mayor porcentaje de la población económicamente activa - PEA, en especial la no calificada, generando adicionalmente divisas para el ahorro, o como capital de inversión, un fuerte encadenamiento y asegura el acceso de alimentos dentro del país, uno de los principios de la seguridad alimentaria.

65 Vol. 4, núm. 2, abril, 2018, pp. 63-80

Víctor Quinde Rosales, Rina Bucaram Leverone, Martha Bucaram Leverone, Francisco Quinde Rosales 
Dom. Cien., ISSN: 2477-8818

Vol. 4, núm. 2, abril, 2018, pp. 63-80

La agricultura se encuentra ligada al crecimiento y desarrollo de la nación republicana en términos históricos, sociológicos y económicos, es bajo esta importancia que el mencionado sector necesita mantener su progreso productivo, el cual es importante y vinculante para el desarrollo económico y social de la población del Ecuador. Para el logro de la mencionada premisa, la agricultura debe presentar una estrecha relación entre su desarrollo y los medios de inversión y financiamiento que generen un factor productivo para beneficio del sector y del Ecuador. En este estudio se analiza la contribución que genera el gasto público, la inversión extranjera directa y el crédito de la banca, entendiendo que estas variables son las fuentes de inversión y financiamiento para el desarrollo productivo del sector, manifestado en el Producto Interno Bruto - PIB agrícola.

Asimismo, se caracteriza la importancia que tiene el gasto público, la inversión extranjera directa y el crédito de la banca en la producción agrícola mediante la revisión de la literatura de autores que han caracterizado a estas fuentes de inversión y financiamiento, para luego establecer el marco metodológico que engloba el desarrollo del estudio, posteriormente los resultados de la investigación se manifestarán ante la presentación del modelo de regresión múltiple que evidencie la contribución de las variables antes mencionadas.

\section{Desarrollo}

Naranjo (2009), manifiesta la relación existente entre la independencia del territorio ecuatoriano del yugo español y el florecimiento agrícola en términos productivos y comerciales bajo el estandarte de la producción cacaotera que tenía esta población limitado por la institucionalidad colonial que buscaba un monopolio. Las teorías de David Ricardo (2009), promovieron un libre comercio en los puertos Iberoamericanos llevando a Guayaquil a desarrollar la idea de crear una nación desde este puerto principal.

Es el cacao conocido para la época como "pepa de oro" el que no solo generó el impulso libertario, sino que también según Acosta (2006), generó implicaciones económicas y sociales convirtiéndose en el impulso y recuperación económica del Ecuador en los inicios de la república, desarrollando un

66 Vol. 4, núm. 2, abril, 2018, pp. 63-80

Víctor Quinde Rosales, Rina Bucaram Leverone, Martha Bucaram Leverone, Francisco Quinde Rosales 
Dom. Cien., ISSN: 2477-8818

Vol. 4, núm. 2, abril, 2018, pp. 63-80

Inversión y financiamiento para el sector agrícola del Ecuador: aplicación de un modelo de regresión múltiple

auge económico a fines del siglo XIX gracias a que este producto produjo el principal vínculo de integración del Ecuador en el mercado mundial.

Actualmente, la agricultura presenta una importancia en el desarrollo económico del país, entendiendo que este no se encuentra aislado del resto de sectores. Hirschman (1958) y Arias et al. (2005) coinciden en que la agricultura sigue siendo aún el ente de desarrollo económico más importante que el del resto de sectores. Al mismo tiempo, Hayami et al. (1985) describe al primer factor como la interrelación de la agricultura e independencia de la misma con el resto de la economía. La Organización de la Naciones Unidas para la Alimentación y la Agricultura - FAO (1995), complementa al decir que, para lograr el crecimiento económico de un país, la agricultura debe aportar articuladamente con el resto de sectores para maximizar el beneficio de las ventajas competitivas y acumular capital.

Por su parte, la Comisión Económica para América Latina y el Caribe - CEPAL (2001), describe al segundo factor como la influencia que tiene la agricultura en el medio de vida rural y la soberanía alimentaria y que para corregir la pobreza de sus pobladores es necesario el desarrollo de la actividad económica de los territorios rurales generando una agricultura dinámica y competitiva que genere empleo y a su vez una demanda de servicios y productos que dinamice la economía.

Echeverri et al. (2002), citado por FAO (2004), mencionan como tercer factor la capacidad de la agricultura al abordar dimensiones económicas y no económicas que en la actualidad son prioritarias para todos, entendiendo que la agricultura genera un conjunto de externalidades, las cuales pueden ocasionar beneficios o perjuicios a la actividad productiva y que se escapan de la misma pero que afectan al conjunto social.

Quinde (2015), manifiesta que estos tres factores demuestran que el sector agrícola no solo es la actividad primaria productiva, el Ecuador debe tomar al sector como un activo estratégico. Yannuzzelli (2017), menciona que el sector agrícola es el eje vinculante de una economía en vía de desarrollo, por lo tanto, es menester generar inversión o financiación que conlleve al progreso de este sector. Para conservar y mejorar a este sector es necesario establecer y promover fuentes de inversión y financiamiento que de forma recíproca contribuyan al desarrollo productivo del mencionado sector;

67 Vol. 4, núm. 2, abril, 2018, pp. 63-80

Víctor Quinde Rosales, Rina Bucaram Leverone, Martha Bucaram Leverone, Francisco Quinde Rosales 
Dom. Cien., ISSN: 2477-8818

Vol. 4, núm. 2, abril, 2018, pp. 63-80

Inversión y financiamiento para el sector agrícola del Ecuador: aplicación de un modelo de regresión múltiple

reconociendo la funcionalidad que existe entre la agricultura y los otros sectores en especial la industria. La FAO (1996), nombra a dos fuentes de inversión o de financiamiento principales en Latinoamérica, dichas fuentes son la pública y la privada.

Yannuzzelli et al. (2017) concibe al gasto público como la inversión que realiza el gobierno para lograr un desarrollo en el país, donde se priorizan las necesidades de los sectores que componen la economía, obligaciones inmediatas del gobierno como programas y proyectos que generen un beneficio a la sociedad y a los sectores económicos que la componen.

Al respecto, la FAO (2007) agrega que el gasto público representa el esfuerzo de un país para atender necesidades nacionales prioritarias y financiar programas fundamentales de la política de desarrollo. Barcelata (2007) sugiere que el gasto público debe ser considerado como un factor de impulso al desarrollo que asegura el funcionamiento adecuado de la economía y garantiza el bienestar social, es bajo esta premisa que existe la necesidad de incrementarlo, tal como lo hacen los países más desarrollados.

Esta idea es ratificada por Hall (2010), el cual manifiesta que a pesar de que el gasto público sea considerado como una carga para una economía de mercado la cual crecería más rápido si se limitara el mencionado gasto, la historia demuestra lo contrario, desde mediados del siglo XIX el crecimiento económico ha presentado una estrecha relación con el incremento proporcional del gasto público.

Guereña (2011), sostiene que el gasto público es la expresión más clara de la voluntad política entendiendo que la elaboración y ejecución del presupuesto del estado implica asignar prioridades entre las diferentes necesidades de inversión que tienen los sectores los cuales compiten por recursos limitados. A esta aseveración Quinde (2011), agrega que el estado ecuatoriano debe tener en cuenta la importancia que tiene el sector agrícola en términos de producción para el consumo interno y de su contribución a la economía con las exportaciones antes de beneficiar a otros sectores económicos del país.

Ramírez (2016), manifiesta como postura teórica el argumento keynesiano que describe la capacidad que tiene el gasto de gobierno para generar un efecto positivo sobre la actividad económica dejando

68 Vol. 4, núm. 2, abril, 2018, pp. 63-80

Víctor Quinde Rosales, Rina Bucaram Leverone, Martha Bucaram Leverone, Francisco Quinde Rosales 
Dom. Cien., ISSN: 2477-8818

Vol. 4, núm. 2, abril, 2018, pp. 63-80

Inversión y financiamiento para el sector agrícola del Ecuador: aplicación de un modelo de regresión múltiple

de lado brechas existentes entre la actividad potencial y la real generando una mayor recaudación impositiva, estableciendo al gasto como un generador de ingresos.

A pesar de ello Teruel et al. (2004) expone que en Latinoamérica entre 1980 y 1990 los sistemas financieros de fomento al desarrollo agrícola fueron muy cuestionados por su funcionamiento ineficiente y politizado. Sin embargo, Ramírez $(2014,2016)$ expresa que la diferencia entre finanzas públicas y privadas se manifiesta en que las finanzas privadas son ingresos disponibles que buscan ingresos futuros, generándose como problema la aplicación del recurso para la búsqueda de mencionados ingresos, las finanzas públicas son gastos determinados por las necesidades de la sociedad y en función de esto obtendrán los recursos buscando el beneficio para la sociedad.

La inversión extranjera directa - IED es una de las fuentes de financiamiento consideradas para el desarrollo del sector agrícola. Concha et al. (2016) expone que la globalización profundizó la creciente interdependencia de los países, gracias a la fuerte expansión de comercio, capital y tecnología internacional. Chudnovsky (2007), citado por el mismo Concha et al. (2016), corrobora la premisa anterior indicando que el IED obtuvo un incremento en la última década del siglo XX sin precedentes a nivel mundial.

Obeid (2015), explica que la IED beneficia a los países receptores no solo con el ingreso de capitales, sino con conocimientos tecnológicos, desarrollo del capital humano y capacidad de gestión necesaria para el desarrollo económico sostenible de los sectores beneficiarios. Zapata (2007) es directa al manifestar la importancia que de la IED y el dinamismo que generan estas empresas en los sectores económicos de América Latina. Kok et al. (2009) manifiesta el desconocimiento en términos de establecer que variables explicativas son determinantes para el desarrollo de la IED. Felisoni et al. (2010) menciona la heterogeneidad de los determinantes que inciden en la IED según las regiones mundiales.

Ante esto Anderson et al. (1986) y Agarwal et al. (1992) establecen la existencia de una complejidad al momento de generarse la IED, esto debido al riesgo, control y compromiso de sus recursos. Deresky

69 Vol. 4, núm. 2, abril, 2018, pp. 63-80

Víctor Quinde Rosales, Rina Bucaram Leverone, Martha Bucaram Leverone, Francisco Quinde Rosales 
Dom. Cien., ISSN: 2477-8818

Vol. 4, núm. 2, abril, 2018, pp. 63-80

Inversión y financiamiento para el sector agrícola del Ecuador: aplicación de un modelo de regresión múltiple

(2000) citado por Zapata (2007) argumenta que el desarrollo de la IED trae consigo costos muy altos, pero ofrece mayor control y flexibilidad.

Según Felisoni et al. (2010) citado por Concha et al. (2016), los factores sociopolíticos, institucionales, tamaño del mercado, el PIB, las exportaciones y el riesgo país pueden considerarse como variables a tomar en cuenta para determinar donde se da la IED. Kok et al. (2009), García et al. (2008) citado por Concha et al. (2016) y Dauti (2015) agregan que en los mercados emergentes la IED busca un ambiente económico transparente de parte del estado y las empresas por encima de los incentivos que se puedan generar.

O’Meara (2015) describe a la IED como el mecanismo de ingreso de capital más idóneo gracias a sus beneficios en términos de crecimiento a largo plazo y desarrollo económico, ya que el país anfitrión puede obtener la ventaja de adaptar y emular la tecnología y gestión extranjera, adicionalmente generan empleo, productividad y desarrollo económico.

La última de las fuentes de inversión o financiamiento a estudiar es el crédito bancario, Quinde et al. (2017) menciona que la banca es uno de los principales actores dentro de una economía al encargarse de impulsar el desarrollo económico, mediante la asignación de recursos para ser invertidos y generar una economía más dinámica.

Clavellina (2013) expone que una economía en desarrollo debe ver con buenos ojos al crédito bancario ya que este se vuelve en un elemento esencial para el apoyo a sus actividades productivas. Honohan (2004) reconoce la existencia de un vínculo positivo entre el financiamiento y el crecimiento entendiendo que es necesario mencionado crecimiento para reducir los niveles de pobreza de una nación.

Adicional a lo mencionado Pagano (1993), expresa que los intermediarios financieros incrementan la productividad generando una relación sobre el crecimiento económico. Mckinnon (1973), Shaw (1973) y Warman et al. (1994) establecen que es necesario una liberalización financiera que genere beneficios para el desarrollo económico de un país.

70 Vol. 4, núm. 2, abril, 2018, pp. 63-80

Víctor Quinde Rosales, Rina Bucaram Leverone, Martha Bucaram Leverone, Francisco Quinde Rosales 
Dom. Cien., ISSN: 2477-8818

Vol. 4, núm. 2, abril, 2018, pp. 63-80

Hernández (2009), llega a comparar al gasto publico productivo con la inversión privada manifestando que son complementarios, sustitutos o independientes uno del otro. Quinde (2017) expresa el interés mostrado por el estado ecuatoriano en desarrollar el sistema financiero, pero al mismo tiempo presenta una complejidad al ser vinculante con un sector socio-productivo como el agrícola. Es por esto por lo que no solo es necesario generar mayor cantidad de desembolsos crediticios sino también como dar vínculo al pequeño productor con el sistema bancario.

Ayala et al. (2014) confirma lo expuesto manifestando que existen diversas teorías que exponen la importancia del crédito en el crecimiento económico de los países, a pesar de ello la mayor o menor contribución de éste, gira en función de las condiciones de cada una de las economías.

Díaz (2014) analiza esta interrogante citando a Hamilton (1781) y su argumento de que los bancos son los motores que el hombre pudo inventar para buscar el crecimiento económico; mientras que Adams (1819) manifiesta que los bancos son nocivos para la moral y la tranquilidad humana e incluso distorsionan la riqueza de las naciones. Finalmente, Díaz (2014) describe que actualmente la teoría económica establece que el financiamiento interno es una condición fundamental para el crecimiento económico.

\section{Metodología}

El artículo presenta un tipo de razonamiento inductivo el cual, usa un modelo econométrico para medir la probabilidad de los argumentos establecidos que determinen la contribución de las variables que generan inversión o financiamiento en la producción agrícola manifestada en el PIB. Se esboza como proceso de desarrollo de la investigación un proceso unimétodo con un paradigma empírico-analítico según Bacon (1949) citado por Duque (2015) permite reflejar la realidad de la forma más fiel y neutral posible de la investigación realizada.

El diseño de investigación del documento estableció una base de datos de serie de tiempo trimestral con un periodo de evaluación del primer trimestre del 2005 al cuarto trimestre del 2015 de las variables homologadas PIB agrícola, gasto público - desarrollo agropecuario, inversión extranjera directa

71 Vol. 4, núm. 2, abril, 2018, pp. 63-80

Víctor Quinde Rosales, Rina Bucaram Leverone, Martha Bucaram Leverone, Francisco Quinde Rosales 
Dom. Cien., ISSN: 2477-8818

Vol. 4, núm. 2, abril, 2018, pp. 63-80

agrícola, crédito agrícola público, crédito agrícola privado, y el generado por otras financieras; cuya base fue obtenida del Banco Central del Ecuador - BCE y la Superintendencia de Bancos y Seguros - SBS. La propuesta metodológica de la investigación propone un modelo de regresión múltiple que establezca los coeficientes de regresión parcial que demuestran la contribución de las variables independientes.

Gujarati y Porter (2010) manifiesta que el método de mínimos cuadrados ordinarios del matemático alemán Carl Friedrich Gauss, se considera como el modelo clásico o estándar de regresión lineal MCRL, siendo este la base de la teoría econométrica, sobre la cual se generan los modelos de regresión simple y de regresión múltiple, modelo en los cuales hay más de una variable explicativa o regresora.

Generalizando la función de regresión poblacional (FRP):

$$
\text { (1) } Y_{t}=\beta_{0}+\beta_{1} X_{1}+\beta_{2} X_{2}+\beta_{3} X_{3}+\beta_{4} X_{4}+\beta_{5} X_{5}+\mu_{t}
$$

Para efectos de simetría notacional:

$$
\text { (1) } Y_{t}=\beta_{1} X_{1}+\beta_{2} X_{2}+\beta_{3} X_{3}+\beta_{4} X_{4}+\beta_{5} X_{5}+\mu_{t}
$$

$Y$ es la variable dependiente, $X_{1}, X_{2}, X_{3}, X_{4}$ y $X_{5}$ son las variables explicativas, $\beta_{1}, \beta_{2}, \beta_{3}, \beta_{4}$ y $\beta_{5}$ se denominan coeficiente de regresión parcial, $\mu$ es el término de perturbación estocástica, y t es la $t$ ésima observación de serie de tiempo.

\section{Resultados}

Para medir la contribución de las fuentes de inversión y financiamiento en el desarrollo productivo del sector agrícola, se realizó una primera función en la cual se tomaron como variables independientes al Gasto Publico Agrícola, IED al Sector Agrícola, Banca Privada, Banca Pública y otras Financieras que caracteriza a Mutualistas, Sociedades Financieras y Corporaciones de Titularización Hipotecaria; mientras que, como variable dependiente al PIB Agrícola, obteniendo el siguiente modelo:

72 Vol. 4, núm. 2, abril, 2018, pp. 63-80

Víctor Quinde Rosales, Rina Bucaram Leverone, Martha Bucaram Leverone, Francisco Quinde Rosales 
Dom. Cien., ISSN: 2477-8818

Vol. 4, núm. 2, abril, 2018, pp. 63-80

Inversión y financiamiento para el sector agrícola del Ecuador: aplicación de un modelo de regresión múltiple

(1) $P I B=f($ Gasto Público + Inversión Extranjera Directa + Banca Privada + Banca Pública + Otras Finacieras)

El PIB Agrícola muestra una pendiente positiva dentro del periodo estudiado, comportamiento heterogéneo a la irregular tendencia que presentan las variables Gasto Publico Agrícola, IED al Sector Agrícola, Banca Privada, Banca Pública y otras Financieras que generan crédito al sector agrícola las cuales presentan notorios puntos críticos máximos y mínimos lo que denota lo susceptible que es el sector a desinversiones o bajas de financiamiento a pesar de su constante incremento productivo. (Figura 1).

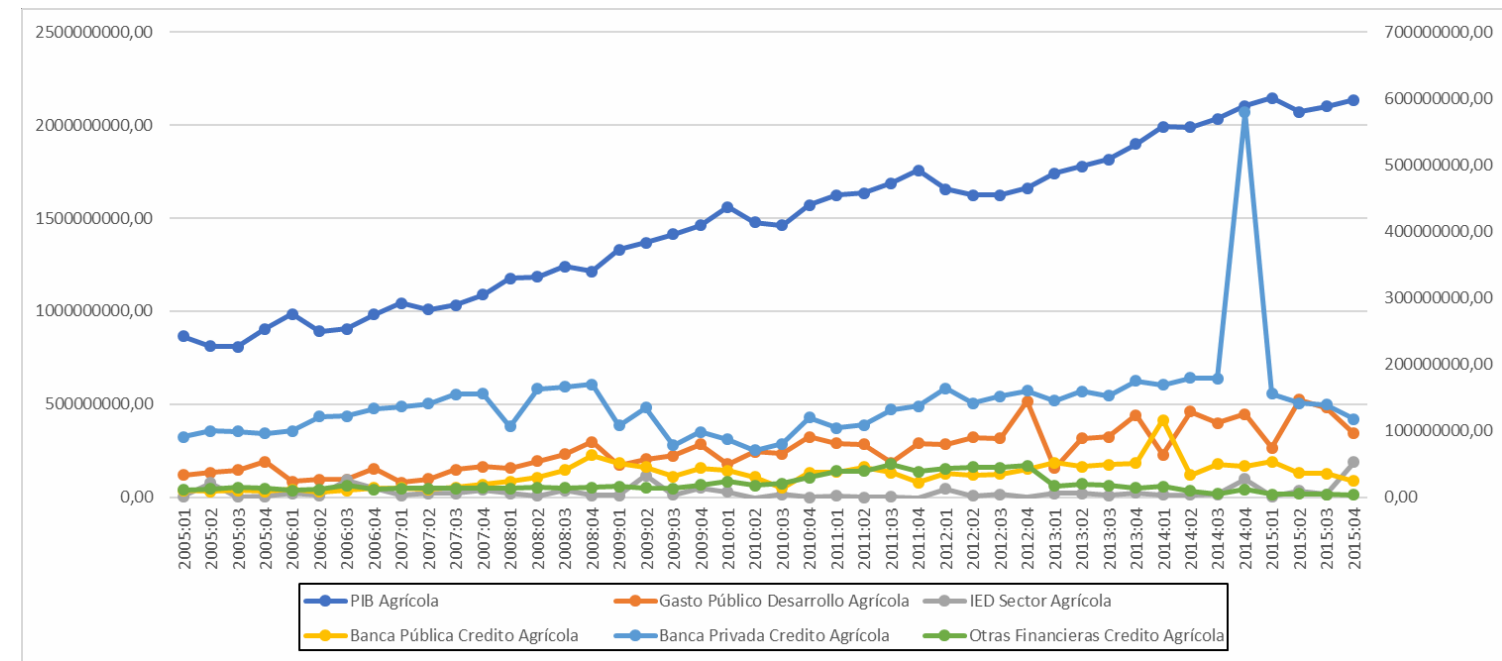

Figura. 1. PIB Agrícola, Gasto Publico Agrícola, IED al Sector Agrícola, Banca Privada, Banca Pública y otras Financieras

El mencionado modelo favorece el resultado de los valores obtenidos en $\mathrm{R}^{2}$ logrando una mayor cobertura en términos de la capacidad explicativa, Prob (F-statistic) el cual busca una reducción de la probabilidad de cometer el error de tipo I y la no existencia de correlación serial que evalúa el test de Durbin-Watson al momento de determinar una independencia de los datos; poniéndolos a estos por encima de los valores obtenidos en los test de Akaike info criterion y Schwarz criterion los cuales presentan un valor significativamente alto (Tabla 1). 
Dom. Cien., ISSN: 2477-8818

Vol. 4, núm. 2, abril, 2018, pp. 63-80

Inversión y financiamiento para el sector agrícola del Ecuador: aplicación de un modelo de regresión múltiple

Tabla 1. Estimación de Parámetros y Pruebas Estadísticas

\begin{tabular}{ccccc}
\hline \hline $\mathbf{R}^{\mathbf{2}}$ & Prob (F-statistic) & Durbin-Watson & Akaike & Schwarz \\
\hline 0.757529 & 0.000000 & 1.495332 & 41.36242 & 41.60572 \\
\hline \hline
\end{tabular}

La matriz de correlación parcial demuestra la no existencia de multicolinealidad ya que las variables independientes estudiadas no están estrechamente relacionadas, presentando ninguna de ellas un valor mayor al 0,8 (Tabla 2).

Tabla 2. Matriz de Correlación Parcial

\begin{tabular}{ccccccc}
\hline \hline & PIB & GASTO_PUBLICO & IED & BANCA_PUBLICA & BANCOS_PRIVADOS & OTRAS_FINANCIERAS \\
\hline PIB & 1 & & & & & \\
GASTO_PUBLICO & 0.797228 & 1 & & & & \\
IED & 0.087714 & 0.024844 & 1 & & 1 & 1 \\
BANCA_PUBLICA & 0.637965 & 0.421786 & -0.084096 & 0.273267 & 1 & -0.078363 \\
BANCOS_PRIVADOS & 0.368903 & 0.392313 & 0.259705 & 0.096574 & & 1 \\
OTRAS_FINANCIERAS & 0.101394 & 0.119740 & -0.303394 & 0.09634 & \\
\hline \hline
\end{tabular}

El test White corrobora la aseveración que muestra, que el modelo estudiado es homocedástico, es decir que las perturbaciones tienen la misma varianza, y esta es constante para las diferentes regresoras, (Tabla 3).

Tabla 3. Test de White

\begin{tabular}{lllr}
\hline \hline Heteroskedasticity Test: White & & & \\
\hline F-statistic & 1.897345 & Prob. F(20,23) & 0.0704 \\
Obs*R-squared & 27.39538 & Prob. Chi-Square(20) & 0.1245 \\
Scaled explained SS & 29.37328 & Prob. Chi-Square(20) & 0.0807 \\
\hline \hline
\end{tabular}

Para analizar la autocorrelación serial el test de Breusch-Godfrey Serial Correlation LM demuestra que sin la necesidad de agregar rezagos el modelo está libre de autocorrelación encontrando una explicación en el pasado (Tabla 4). 
Dom. Cien., ISSN: 2477-8818

Vol. 4, núm. 2, abril, 2018, pp. 63-80

Inversión y financiamiento para el sector agrícola del Ecuador: aplicación de un modelo de regresión múltiple

Tabla 4. Breusch-Godfrey Serial Correlation LM

\begin{tabular}{llll}
\hline \hline \multicolumn{3}{l}{ Breusch-Godfrey Serial Correlation LM Test: } & \\
\hline F-statistic & 2.973809 & Prob. F(1,37) & 0.0930 \\
Obs*R-squared & 3.273334 & Prob. Chi-Square(1) & 0.0704 \\
\hline \hline
\end{tabular}

Se establece como modelo final PIB $=\mathrm{f}($ Gasto Público + IED + Banca Privada + Banca Pública + Otras Financieras), el cual en términos de sustitución de coeficientes genera el resultado.

$$
\mathrm{PIB}=7.83793412924 * \text { GASTO_PUBLICO }+4.57429570437 * \text { IED }+
$$

\subsection{7*BANCA_PUBLICA - 0.0702660062717*BANCOS_PRIVADOS +} $0.723845266619 *$ OTRAS_FINANCIERAS +614757386.16

Los resultados muestran un análisis e interpretación dentro del rango ordinal al no presentar alteraciones en las variables que forman parte de mencionado modelo. La banca privada no contribuye directamente en el desarrollo del PIB Agrícola, a diferencia de la Banca Pública, el Gasto Público y el IED quienes son dentro de ese orden las variables de mayor contribución a la producción agrícola.

\section{Conclusiones}

El sector agrícola presenta una estrecha e importante relación con la creación de la república ecuatoriana, el desarrollo social y económico de su población y demás sectores. Mencionado sector debe recibir fuentes de inversión y financiamiento recíprocos a su aporte en la economía ecuatoriana.

Las fuentes más importantes para generar una inversión o financiamiento de la agricultura son el gasto público, la inversión extranjera directa y el crédito bancario público y privado, convirtiéndose estas con sus capitales en impulso para el crecimiento de este sector histórico. A pesar de ello la contribución de las mismas son irregulares y flexibles al proceso de inversión.

La agricultura encuentra su mayor aporte en la banca pública, la misma que tiene la capacidad de impulsar la producción agrícola en casi ocho veces su inversión, del mismo modo el gasto público

75 Vol. 4, núm. 2, abril, 2018, pp. 63-80

Víctor Quinde Rosales, Rina Bucaram Leverone, Martha Bucaram Leverone, Francisco Quinde Rosales 
Dom. Cien., ISSN: 2477-8818

Vol. 4, núm. 2, abril, 2018, pp. 63-80

Inversión y financiamiento para el sector agrícola del Ecuador: aplicación de un modelo de regresión múltiple

para el desarrollo agrícola y los créditos otorgados por otras financieras contribuyen con cantidades muy similares al progreso productivo del sector agrícola.

La variable banca privada no contribuye directamente en la formación del PIB Agrícola, ratificando las teorías de que no por generar mayor cantidad de préstamos estos harán más productivo al sector.

La inversión extranjera directa contribuye a la agricultura, pero en menor proporción que el crédito de la banca pública, el gasto público y el crédito de otras financieras esto debido a la poca orientación de capitales extranjeros en mencionado sector.

\section{Referencias Bibliográficas}

ACOSTA, A. Breve Historia Económica del Ecuador. Ecuador: Editora Nacional. 2006.

ADAMS, J. Quoted from Hammond, Banks and Politics in America: From the Revolution to the Civil War. Princeton: Princeton University Press. 1819.

AGARWAL, S Y RAMASWAMI, S. 1992. Choice of foreing market entry modes: Impact of ownership, Location and internalization factors. Journal of International Business Studies. First Quarter. Pp. 1-26.

ANDERSON, E Y GATIGNON, H. 1986. Modes of foreing entry: A transaction cost analysis and propositions. Journal of International Business Studies. Vol. 17. Pp. 1-26.

ARIAS, J; VALLEJO, S Y TREJOS, R. La Real Contribución de la Agricultura a la Economía del Ecuador. Ecuador: Instituto Interamericano de Cooperación para la Agricultura - IICA. 2005.

AYALA, D; MENA, G Y MARAVILLA, F. Contribución del Crédito Bancario al Crecimiento Económico en El Salvador, período 2004-2013. San Salvador: Universidad de El Salvador. 2014.

BARCELATA, H. Las Finanzas Públicas En México. 1988-2006. México: Eumed. 2007.

CEPAL, Comisión Económica para América Latina y el Caribe. Revalorar la agricultura y el desarrollo rural para la sustentabilidad. México. 2001.

76 Vol. 4, núm. 2, abril, 2018, pp. 63-80

Víctor Quinde Rosales, Rina Bucaram Leverone, Martha Bucaram Leverone, Francisco Quinde Rosales 
Dom. Cien., ISSN: 2477-8818

Vol. 4, núm. 2, abril, 2018, pp. 63-80

Inversión y financiamiento para el sector agrícola del Ecuador: aplicación de un modelo de regresión múltiple

CLAVELLINA, J. Crédito bancario y crecimiento económico en México. Mexico: Economía Informa. 2013.

CONCHA, J Y GÓMEZ, O. Análisis de atracción de inversión extranjera a países de la Alianza del Pactico. España: Estudios Gerenciales. 2016.

DAUTI, B. Determinants of foreing direct investment in south east european countries and new member states of European Union Countries. Economic and Business Review. 2015.

DÍAZ, V. Crédito privado, crédito bancario y producto interno bruto: evidencia para una muestra suramericana. España: Ensayos sobre Política Económica. 2014.

DUQUE, E. Seminario Metodología de la Investigación. Colombia: Universidad Nacional de Colombia. 2015.

FAO, Organización de las Naciones Unidas para la Alimentación y la Agricultura. El Estado Mundial de la Agricultura y la Alimentación. Roma. 1995.

FAO, Organización de las Naciones Unidas para la Alimentación y la Agricultura. Política de desarrollo agrícola. Roma. 2004.

FAO, Organización de las Naciones Unidas para la Alimentación y la Agricultura. La inversión en la agricultura: evolución y perspectivas. Roma: Banco Mundial - BM. Cumbre Mundial Sobre La Alimentación. 1996.

FAO, Organización de las Naciones Unidas para la Alimentación y la Agricultura. Análisis Integral Del Gasto Público Agropecuario. México. 2007.

FELISONI, C; EUNNI, R Y MARTINS, N. 2010. Determinants of FDI in Emerging Markets: Evidence from Brazil. Journal of Commerce and Management. Vol. 20. Pp. 203-216.

GUEREÑA, A. Derecho a producir. Invertir más y mejor en la pequeña agricultura de América del sur. Mexico: Oxford Committee for Famine Relief - Oxfam. 2011.

77 Vol. 4, núm. 2, abril, 2018, pp. 63-80

Víctor Quinde Rosales, Rina Bucaram Leverone, Martha Bucaram Leverone, Francisco Quinde Rosales 
Dom. Cien., ISSN: 2477-8818

Vol. 4, núm. 2, abril, 2018, pp. 63-80

Inversión y financiamiento para el sector agrícola del Ecuador: aplicación de un modelo de regresión múltiple

GUJARATI, D Y PORTER D. Econometría. México: The McGraw-Hill. 2010.

HALL, D. Por qué es necesario el gasto público. Reino Unido: Public Services International - PSI, Business School, University of Greenwich. 2010.

HAYAMI, Y y VERNON, R. Agricultural Development: An International Perspective. Baltimore: Johns Hopkins University Press. 1985.

HERNÁNDEZ, J. La composición del gasto público y el crecimiento económico. México: Revista Análisis Económico. 2009.

HIRSCHMAN, A. The Strategy of Economic Development. New Haven: Yale University Press. 1958.

HONOHAN, P. Financial development, growth and poverty: how close are the links?, Policy research working paper. Banco Mundial. 2004.

KOK, R. Y ACIKGOZ, B. 2009. Analyses of FDI determinants in developing countries. International Journal of Social Economics. Vol. 36, Pp. 105-123.

MCKINNON, R. Money and Capital in Economic Development. Washington DC.: The American Political Science Review. 1973.

NARANJO, M. 2009. Un Puerto en busca de una Nación, Guayaquil y la idea fundacional del Ecuador como país. Ecuador: Facultad Latinoamericana de Ciencias Sociales - FLACSO. Seminario Internacional Poder, Política y Repertorios de la Movilización Social en el Ecuador Bicentenario.

O'MEARA, G. 2015. Examining the determinants of foreing direct investment. Undergraduate Economic Review. Vol. 11.

OBEID, A. 2015. The determinants of foreing Direct Investment - Empirical evidence from bahrain. Estados Unidos: International Journal of Business and Social Science. Vol. 6, No. 8, Pp. 94-106.

78 Vol. 4, núm. 2, abril, 2018, pp. 63-80

Víctor Quinde Rosales, Rina Bucaram Leverone, Martha Bucaram Leverone, Francisco Quinde Rosales 
Dom. Cien., ISSN: 2477-8818

Vol. 4, núm. 2, abril, 2018, pp. 63-80

Inversión y financiamiento para el sector agrícola del Ecuador: aplicación de un modelo de regresión múltiple

PAGANO, M. Financial Markets and the Macroeconomy. Financial markets and growth An overview. European Economic Review. 1993.

QUINDE-ROSALES, F. Modelo de Regresión Lineal, para medir la incidencia del sector Bancario en el sector Agrícola primario ecuatoriano Periodo 2005 - 2015. Ecuador: Universidad Agraria del Ecuador - UAE. 2017.

QUINDE-ROSALES, F; BUCARAM-LEVERONE, R Y QUINDE-ROSALES, V. 2017. Aporte del Crédito Público al Sector Agrícola. Una Aplicación del Índice de Orientación Agrícola (IOA). Ecuador: Universidad Agraria del Ecuador - UAE. $\mathrm{I}^{\circ}$ Congreso Estudiantil de Ciencias Agrarias

QUINDE-ROSALES, V. Efecto de la Intervención Estatal en la Regulación de los Precios del Arroz Cantón Daule. Ecuador: Universidad Agraria del Ecuador - UAE. 2015.

QUINDE-ROSALES, V. Evolución del Gasto Público Agropecuario y Rural en el Ecuador Periodo 2000-2009. Ecuador: Universidad Agraria del Ecuador - UAE. 2011.

RAMÍREZ, E. Causalidad entre ingresos y gastos públicos en México (1982-2015). México: universidad Nacional Autónoma de México. 2016.

RAMÍREZ, E. Elementos, Disyuntivas y Discusiones Básicas en el Debate de las Finanzas Públicas. México: Mundo Siglo XXI. 2014.

SHAW, E. Financial Deepening in Economic Development. Nueva York: Oxford University Press. 1973.

TERUEL, R Y OLGUIN, M. Inversión y Financiamiento para la Agricultura y el Desarrollo Rural en la Región Andina. Bolivia: Instituto Interamericano de Cooperación para la Agricultura - IICA. 2004.

WARMAN, F Y THIRLWALL, A. 1994. Interest rate, savings, investment and growth in México, 1960-1990: Tests of financial liberalization hypothesis, Journal of Development Studies

79 Vol. 4, núm. 2, abril, 2018, pp. 63-80

Víctor Quinde Rosales, Rina Bucaram Leverone, Martha Bucaram Leverone, Francisco Quinde Rosales 
Dom. Cien., ISSN: 2477-8818

Vol. 4, núm. 2, abril, 2018, pp. 63-80

Inversión y financiamiento para el sector agrícola del Ecuador: aplicación de un modelo de regresión múltiple

YANNUZZELLI, J. El Índice de Orientación Agrícola (IOA) como Mecanismo del Análisis del Gasto Público Agropecuario Ecuatoriano. Período 2005-2015. Ecuador: Universidad Agraria del EcuadorUAE. 2017. 